\title{
Admission Control over Internet of Vehicles attached with Medical sensors for Ubiquitous Healthcare Applications
}

\author{
Di Lin ${ }^{1}$, Fabrice Labeau ${ }^{2}$, Yuanzhe Yao $^{1}$, Athanasios V.Vasilakos ${ }^{3}$, Yu Tang ${ }^{1}$ \\ ${ }^{1}$ School of Information and Software Engineering, University of Electronic Science and Technology of China \\ ${ }^{2}$ Department of Electrical and Computing Engineering, McGill University, Canada \\ ${ }^{3}$ Department of Computer and Telecommunications Engineering, University of Western Macedonia, Greece
}

\begin{abstract}
Wireless technologies and vehicle-mounted or wearable medical sensors are pervasive to support ubiquitous healthcare applications. However, a critical issue of using wireless communications under a healthcare scenario rests at the electromagnetic interference (EMI) caused by radio frequency (RF) transmission. A high level of EMI may lead to a critical malfunction of medical sensors, and in such a scenario, a few users who are not transmitting emergency data could be required to reduce their transmit power or even temporarily disconnect from the network in order to guarantee the normal operation of medical sensors as well as the transmission of emergency data. In this paper, we propose a joint power and admission control algorithm to schedule the users' transmission of medical data. The objective of this algorithm is to minimize the number of users who are forced to disconnect from the network while keeping the EMI on medical sensors at an acceptable level. We show that a fixed point of proposed algorithm always exists, and at the fixed point, our proposed algorithm can minimize the number of low-priority users who are required to disconnect from the network. Numerical results illustrate that the proposed algorithm can achieve robust performance against the variations of mobile hospital environments.
\end{abstract}

Index Terms-Wireless Network, E-health, Internet of Vehicles, Priority of Users, Admission Control

\section{INTRODUCTION}

Recent developments in cellular networks (e.g. Universal mobile telecommunication system, UMTS Network) and vehicle-mounted or wearable medical sensors have enabled the innovative application of wireless health anytime and anywhere. However, radio frequency (RF) transmission can result in electromagnetic interference (EMI) on all of medical sensors, and a high level of interference can even cause malfunction of medical sensors and potentially injure patients [1][3]. Thus, the control of interference (e.g. through admission control) is a critical issue to wireless health and should be addressed under the environment of a mobile hospital, which is defined as Internet of vehicles for e-health applications in this paper. So throughout this paper, we alternatively use the terms of mobile hospital and Internet of vehicles for e-health applications.

E-mail address: lindi@uestc.edu.cn, fabrice.labeau@mcgill.ca, yaoyuanzhe@gmail.com, vasilako@ath.forthnet.gr, yutang@uestc.edu.cn. This work is supported by The National Natural Science Funds of China (No. 61370202).
There is a large body of works related to the application of wireless networks to support health service [1]-[4]. Qinghua et al. in [4] address the possibilities of using wireless technologies in a medical environment. However, the potential EMI problem is not discussed in this work. Phond et al. in [1][3] present the issue of EMI under the scenario of a wireless local area network (WLAN) for e-health applications within a hospital, but the technology of WLAN is not applicable to our scenario, in which a mobile hospital covers a large-scaled area (e.g., a city or a town). Thus, the power and admission control algorithms in [1]-[3] cannot be directly used in a mobile hospital environment, in which our work is interested. Admission control algorithms for cellular networks are firstly addressed in [5]. However, these algorithms determine the transmit power and admission of users according to their channel conditions, and do not take the potential EMI impact into account. In such a scenario, a wireless user who stays close to a medical device could be allowed to transmit data at a high level of power if only the user's communication channel is in good condition. However, the radio frequency (RF) transmission at a high level of power would influence the operation of medical devices ${ }^{1}$. Also the abovementioned algorithms assume that all of users are in the same priority. However, in our scenario, we consider two classes of priority of users, i.e. high-priority users who are transmitting emergency data and low-priority users who are transmitting regular data. Under such a scenario, the algorithms in [1]-[5] cannot ensure that the low-priority users will be firstly required to disconnect from the network when causing serious EMI on medical devices, and instead might require high-priority users to disconnect from the network. Such an improper power allocation and admission control by these algorithms [1]-[5] may lead to the malfunction of EMIsensitive medical devices as well as the block of emergencydata transmission, so the aforementioned algorithms cannot be employed under the scenario of a mobile hospital. The

\footnotetext{
${ }^{1} \mathrm{~A}$ few mobile hand-held or body attached devices are functioning in the same RF range, and also may lead to the interference to the other devices. However, most devices transmit data with the technologies of Bluetooth or Zigbee. The typical transmit power of Bluetooth or Zigbee is around $2.5 \mathrm{~mW}$ for the coverage of $10 \mathrm{~m}$, while the typical transmit power of mobile phone is $0.75-1 \mathrm{~W}$. So the interference of medical devices functioning in same RF range can be neglected in comparison with the EMI on medical devices.
} 
importance of scheduling wireless transmission under a mobile hospital scenario as well as the lack of efficient algorithms for power and admission control motivate us to investigate how wireless users can adjust their power to achieve certain goals, such as maximizing the number of users who are allowed to keep connecting to the network while ensuring the minimal amount of EMI on medical devices over Internet of vehicles for e-health applications.

In this paper, we address the problem of dynamically scheduling wireless transmission for wireless users' networks under a mobile hospital environment. The objectives of this paper are to i) minimize the number of users who are required to disconnect from the network; ii) meet certain requirements (e.g., the acceptable level of signal to interference plus noise ratio, SINR) of each user; iii) protect the medical devices from harmful interference. In this paper, we propose a joint power and admission control algorithm in a mobile hospital environment, and the algorithm is shown to converge to a fixed point, at which the minimal number of users who are forced to disconnect from the network is obtained. To the best of our knowledge, this is the first work which presents the joint power and admission control algorithms under a wireless network for e-health applications.

The primary contributions of this paper rest at the following issues:

- Address the framework of data transmission over Internet of vehicles for e-health applications;

- Develop a EMI-based joint power and admission control algorithm which can minimize the EMI caused by RF transmission on medical sensors and minimize the number of users who are required to disconnect from the network to guarantee the transmission of emergency data;

- Investigate how the structure of network impacts the number of users who can keep connecting to the network.

\section{MOBILE HOSPITAL ENVIRONMENT}

A typical mobile hospital environment is composed of vehicles for e-health applications, and these vehicles are mounted with a few medical sensors which can help doctors to monitor the condition of patients. On the vehicle for ehealth applications, doctors, healthcare staff, and the relatives of patients may use mobile phones due to these two issues: (1) Doctors and nurses on the same ambulance must report the conditions of patients over phone to the staff in a hospital or in a medical center to arrange the medical actions which will be taken at the arrival of patients. (2) Patients or their relatives need to contact their family members over mobile phone about the change of clinical situations as well as important information. However, the use of mobile phones may lead to EMI impact on nearby medical sensors [6]. EMI refers to the disturbance of electrical circuits because of either electromagnetic induction or electromagnetic radiation emitted from an external source [1]. The disturbance may degrade the effective performance of the circuit, and the degradationcan lead to a total loss of data. Most problems of performance degradation are due to the component parasitics, and it represents the stray reactive elements which have been found in every component, including both passive and active components. Capacitors have series inductance, which can lead to a series resonant circuit. Wound inductors have interwinding capacitance, which can lead to a parallel resonant circuit. These circuits resonate at the frequencies from $5 \mathrm{MHz}$ to $1000 \mathrm{MHz}$. Besides the issue of component parasitics, the other issues which may lead to the performance degradation of medical devices include ground impedance, poor cable shielding, stray internal coupling paths, etc. [7].

In the following, we first present an experiment to show the effects of EMI on medical sensors and then address the model of EMI impact in this paper as a constraint of outageoptimization problem, which is detailed in Section III.A.

\section{A. Experiment of testing EMI effects}

In this experiment, we test the EMI impact on 50 types of vehicle-mounted and wearable medical sensors from the cellular phones operated by China Mobile, China Unicom, China Telecom. These cellular phones are with the technologies of GSM-900/1800, CDMA2000, TD-LTE. Their average transmit power varies $0.6-1.0 \mathrm{~W}$, their average distance to medical equipments varies from $0.5 \mathrm{~m}-4.5 \mathrm{~m}$, in consideration of the size of an ambulance.

The test is carried out in an anechoic chamber in order to exclude EMI impact from the other sources of RF emission, such as from telecommunication systems. The test procedures are detailed as follows: a) Tabletop sensors are placed on a table $80 \mathrm{~cm}$ above the floor, and floor-standing sensors are placed on the floor; b) One investigator who operates a mobile phone controls the maximal power output $(0.8 \mathrm{~W})$, while another investigator monitors the working status of medical sensors; c) The mobile phone is gradually brought closer to the medical sensor. If the degradation of performance of sensors occurs, the mobile phone is turned off to check if the performance degradation ceases, which shows whether the degradation is reversible or irreversible; d) The EMI impact on medical sensors, reversible or irreversible, as well as the distance between medical sensors and mobile phones at the degradation of performance are recorded.

Test result shows that EMI from cellular phones causes the performance degradation of $68 \%$ of medical sensors within a $2 \mathrm{~m}$ distance away from the cellular phones. Typical degradation in the test includes: a) Artifact in images of ultrasound, Xray, CT sensors; b) Noise on biomedical signals, such as ECG and EEG; c) Sensor malfunction in infusion pumps, syringe pumps, ventilators; d) Change of operating mode of external pacemakers, such as from asynchronized to fixed rate. This result is in line with [1]-[3].

Most problems of performance degradation are due to the component parasitics, and it represents the stray reactive elements which have been found in every component, whether a passive or active component. Capacitors have series inductance, which can lead to a series resonant circuit. Wound inductors have interwinding capacitance, which can lead to a parallel resonant circuit. These circuits resonate at the frequencies from $5 \mathrm{MHz}$ to $1000 \mathrm{MHz}$. Besides the issue of component parasitics, the other issues which may lead to the 
performance degradation of medical sensors include ground impedance, poor cable shielding, stray internal coupling paths, etc. [?], [8]-[10].

\section{B. Model of EMI impact}

A typical vehicle for E-health applications consists of both life-support and non-life-support medical sensors. The medical data which are collected by medical sensors are required to send to the doctors, who are staying in a hospital to make the plan of taking actions on the patient once the vehicle arrives at the hospital. Also the medical staff on the vehicle need to report the condition of patients over phone to doctors, and the use of mobile phone may lead to EMI on medical sensors which are located nearby. The life-support medical sensors contain electronic components which are sensitive to EMI, so they are more sensitive to the impact of EMI than non-life-support sensors. Life-support medical sensors include Ultrasonograph sensors, etc., and non-life-support medical sensors include holters and blood pressure sensors, etc.

Both life-support sensors and non-life-support sensors may have different requirements on the transmit power of a wireless user to ensure that the user's RF transmission causes an acceptable level of EMI on medical sensors. The maximal potential transmit power of each wireless user should satisfy all of these requirements. To the best of our knowledge, Phond et al. in [1] firstly address how to model the EMI effects on medical sensors and calculate the maximal potential transmit power of a wireless user subject to the EMI constraints. Mathematically, the constraints on the transmit power of a wireless user can be shown in equation (1) and equation (2), for life-support medical sensors and non-life-support medical sensors, respectively [1].

$$
\begin{gathered}
\sum_{i \in U} \frac{\mu_{1} \sqrt{P_{i}}}{D_{i}(p)} \leq E_{N L S}(p), \text { for } p \in M_{1} \\
\sum_{i \in U} \frac{\mu_{2} \sqrt{P_{i}}}{D_{i}(q)} \leq E_{L S}(q), \text { for } q \in M_{2}
\end{gathered}
$$

where $E_{N L S}(p)$ and $E_{L S}(q)$ are the acceptable EMI levels for a non-life-support sensor $p$ and a life-support sensor $q$, respectively; $P_{i}$ is the transmit power of a wireless user $i$; $D_{i}(p)$ is the distance between a transmitter of user $i$ and nonlife-support sensor $p$ or life-support sensor $p ; \mu_{1}$ and $\mu_{2}$ are constant, and their values suggested by IEC 60601-1-2 are 7 and 23, respectively [1]. $U$ represents the set of wireless users over the Internet of vehicles. $M_{1}$ represents the set of non-life-support sensors, while $M_{2}$ represents the set of lifesupport sensors.

Let

$$
A=\left(\begin{array}{ccc}
\frac{\mu_{1}}{D_{1}(1)} & \cdots & \frac{\mu_{1}}{D_{n}(1)} \\
\cdots & \cdots & \cdots \\
\frac{\mu_{1}}{D_{1}\left(m_{1}\right)} & \cdots & \frac{\mu_{1}}{D_{n}\left(m_{1}\right)} \\
\frac{\mu_{2}}{D_{1}(1)} & \cdots & \frac{\mu_{2}}{D_{n}(1)} \\
\cdots & \cdots & \cdots \\
\frac{\mu_{2}}{D_{1}\left(m_{2}\right)} & \cdots & \frac{\mu_{2}}{D_{n}\left(m_{2}\right)}
\end{array}\right)
$$

and $x_{i}=\sqrt{P_{i}}$, we can represent (1) and (2) as

$$
A X \leq B
$$

where $X=\left[x_{1}, \cdots, x_{m_{1}}, x_{m_{1}+1}, \cdots, x_{m_{1}+m_{2}}\right]^{T}, B=$ $\left[E_{N L S}(1) \cdots E_{N L S}\left(m_{1}\right), E_{L S}(1) \cdots E_{L S}\left(m_{2}\right)\right]^{T}, m_{1}$ is the cardinality of $M_{1}, m_{2}$ is the cardinality of $M_{2}$.

Remark 2.1: When the number of rows of $A$ equals to $n$, i.e. $m_{1}+m_{2}=n$, then, we can obtain the unique solution $X=A^{-1} B$.

Remark 2.2: When the number of rows of $A$ is less than $n$, i.e. $m_{1}+m_{2}<n$, then, the linear equation is underdetermined. We select the optimal one from infinite solutions subject to the maximization of $\sum_{i \in U} P_{i}$.

Remark 2.3: When the number of rows of $A$ is larger than $n$, i.e. $m_{1}+m_{2}>n$, then, the linear equation is overdetermined. We relax the constraints of (1) and (2) with the best approximation, i.e. $\min _{X}|A X-B|$. So $X=\left(A^{T} A\right)^{-1} A^{T} B$.

Remark 2.4: Given the set of wireless users $U$, the maximal transmit power of any wireless user $i$ (denoted as $\bar{P}_{i}(U)$ ) can ensure that all of medical sensors are free from EMI effects when $m_{1}+m_{2} \leq n$ (see Remark 2.1 and 2.2), and also ensure that the total amount of EMI on medical sensors is minimized when $m_{1}+m_{2}>n$ (see Remark 2.3), since under the latter scenario, the power allocation can ensure $\min _{X}|A X-B|$.

Definition 1: The maximal potential transmit power of user $i$ (i.e. $\bar{P}_{i}$ ) to minimize the total amount of EMI on medical sensors, as obtained from Remark 2.4, is defined as the maximal effective transmit power (METP).

The METP (i.e. $\bar{P}_{i}$ for user $i$ ) will be employed to establish the problem of (8) in III.A., develop the joint power and admission control algorithm in III.B. (see (9)), and address the characteristics of proposed algorithms in III.C. (see Theorem 9).

\section{PROBLEM OF POWER AND ADMISSION CONTROL}

In this section, we first address a few definitions as the preliminaries of power and admission control. Then, we characterize the problem of joint power and admission control.

\section{A. Preliminaries of power and admission control problem}

In this section, we present a few preliminaries for the analysis on joint power and admission control by addressing a few definitions. Under the scenario of healthcare applications, wireless users transmit their medical data, and based on the emergency level of data for transmission, we classify the wireless users into two classes of priority, i.e. high-priority and low-priority users. The basis for classifying the user priority is the emergency level of patients, and the classification of levels can refer to a clinical triage guideline. On an ambulance, the medical team who is accompanying with the patient can decide the priority of patient and can change the patient's priority if this patient's status varies.

Within a cellular network, SINR of wireless user $i$ is defined as the quality of useful information received by user $i$ in the 
presence of interference and noise. Mathematically, SINR of user $i$, denoted as $\theta_{i}$, can be formulated as

$$
\theta_{i}=\frac{W P_{i} h_{i i} / R_{i}}{\sum_{j \neq i} P_{j} h_{j i}+W N_{i}}
$$

where $P_{i}$ denotes the transmit power by user $i ; R_{i}$ denotes the data rate by user $i ; h_{j i}$ denotes the channel condition between user $i$ and user $j ; N_{i}$ denotes the power spectral density of additive white Gaussian noise; $W$ denotes the bandwidth of network.

Lemma 1: Given the level of acceptable SINR $\theta_{i}$ for any $i \in U$ ( $U$ is the set of all of users), the transmit power of each user $P_{i}$ satisfies

$$
P_{i}=f\left(\theta_{i}, U\right) \triangleq \frac{R_{i} \theta_{i} N_{i}}{2 h_{i i}\left(1-\sum_{k \in U} \frac{R_{k} \theta_{k}}{W+R_{k} \theta_{k}}\right)}
$$

Proof: Rewriting (4), we can attain

$$
\theta_{i} R_{i}\left(\sum_{j \neq i} P_{j} h_{j i}+W N_{i}\right)=W P_{i} h_{i i}
$$

Adding $\theta_{i} R_{i} P_{i} h_{i i}$ onto both sides of the above equation, we have

$$
\theta_{i} R_{i}\left(\sum_{j} P_{j} h_{j i}+W N_{i}\right)=P_{i} h_{i i}\left(W+\theta_{i} R_{i}\right)
$$

Let $S_{i}=\sum_{j} P_{j} h_{j i}$, we have

$$
\theta_{i} R_{i}\left(S_{i}+W N_{i}\right)=P_{i} h_{i i}\left(W+\theta_{i} R_{i}\right)
$$

On both sides of the above equation, we sum from $i=1$ to $N$, given $N$ users in the network. Then, we have

$$
W N_{i}+S_{i}=\frac{W N_{i}}{1-\sum_{k} \frac{R_{k} \theta_{k}}{W+R_{k} \theta_{k}}}
$$

Also we rewrite (4) as

$$
R_{i} \theta_{i}=\frac{2 W P_{i} h_{i i}}{S_{i}+W N_{i}}
$$

Substituting (6) into (7), we can obtain (5). The proof follows.

Definition 2: Given the acceptable level of SINR $\hat{\theta}_{i}$ and transmit power $\mathbf{P}$, the set of supported users is defined as

$$
S(\mathbf{P})=\left\{i \mid \theta_{i} \geq \hat{\theta}_{i}\right\}
$$

Remark 3.1: Let $U_{H}$ and $U_{L}$ denote the set of high-priority and low-priority wireless users, respectively. From Definition 1 , the set of supported high-priority users is defined as

$$
S_{H}(\mathbf{P})=\left\{i \mid \theta_{i}(\mathbf{P}) \geq \hat{\theta}_{i} \text { and } i \in U_{H}\right\}
$$

Also, the set of supported low-priority users is defined as

$$
S_{L}(\mathbf{P})=\left\{i \mid \theta_{i}(\mathbf{P}) \geq \hat{\theta}_{i} \text { and } i \in U_{L}\right\} .
$$

Definition 3: Let $U$ denotes the set of all of users. Also $S_{H}^{\prime}(\mathbf{P})=\left\{i \mid \theta_{i}(\mathbf{P})<\hat{\theta}_{i}\right.$ and $\left.i \in U_{H}\right\}$ and $S_{L}^{\prime}(\mathbf{P})=$ $\left\{i \mid \theta_{i}(\mathbf{P})<\hat{\theta}_{i}\right.$ and $\left.i \in U_{L}\right\}$ represent the set of unsupported high-priority and low-priority users, respectively. Then, we define the outage ratio for high-priority users $O_{H}(\mathbf{P})$ and for low-priority users $O_{L}(\mathbf{P})$ as

$$
O_{H}(\mathbf{P})=\frac{\left|S_{H}^{\prime}(\mathbf{P})\right|}{|U|} \text { and } O_{L}(\mathbf{P})=\frac{\left|S_{L}^{\prime}(\mathbf{P})\right|}{|U|}
$$

where $|\cdot|$ represents the cardinality of a set.

In this paper, we are interested in the following problem

$$
\min _{0 \leq \mathbf{P} \leq \overline{\mathbf{P}}} O_{L}(\mathbf{P}) \text { subject to } O_{H}(\mathbf{P})=0
$$

where $\overline{\mathbf{P}}$ represents the METP of power $\mathbf{P}$ in Definition 1 .

Remark 3.2: Problem (8) indicates that we study how to minimize the number of unsupported low-priority users while ensuring that all of high-priority users are supported.

Without the constraints of METP, the optimal admission control algorithm to minimize outage is shown as follows [11]: the low-priority users with the worst channel conditions will be removed first to ensure the minimal outage ratio. However, under the setting of considering METP constraints, the users who violate the EMI constraints must be removed even with a very good channel condition, so the optimal outage ratio can only be achieved in consideration of both channel conditions as well as EMI constraints, increasing the difficulty of finding a feasible solution to the outage-optimization problem of (8).

\section{B. Algorithm of admission control}

We develop our algorithm by referring to a few admission control algorithms in wireless networks, though the latter algorithms are not applicable under the scenario of mobile hospital since these algorithms do not take into account the EMI on medical sensors and multiple classes of priority of users. From the perspective of methodology, the algorithms of admission control in wireless networks are composed of two classes: (1) Performance-tracking (TPC) algorithms [12][14] and (2) Temporary removal and feasibility check (DFC) algorithms [11], [15]-[17].

TPC algorithm is originally proposed by [13], and it is designed to provide all of users with their predefined target SINRs in feasible systems (i.e. no outage) with the minimum consumption of aggregate transmit power. The problem of minimizing the aggregate transmit power is a NP-hard problem, and a few tractable solutions are addressed in [12] and [14]. In TPC algorithms, each user adjusts his/her transmit strategies according to the level of interference from others, and attempts to increase transmit power with the rise of interference level. Thus, this algorithm has the following drawback: a few users may transmit at their maximal level of power while still failing to attain their acceptable level of performance, which leads to a high level of outage ratio.

To avoid the problem of TPC algorithms, a few DFC algorithms are proposed. In [15], any user who exceeds its maximum transmit power in order to reach its target SIR will be permanently removed. When the channel condition becomes acceptable again for these users who are permanently removed, they do not resume their transmission. This is a drawback in a feasible system which becomes infeasible for very short times, since the algorithm causes unnecessary permanent removals. To avoid this problem, Rasti et al. in [16] proposes an algorithm which is revised to temporarily remove a few users who exceed their maximum transmit power in order to reach target SINR. A removed user resumes its transmission when the channel condition turns good again. Yingsong et al. in [17] applies the algorithm in [16] into the 
transmission of video in cellular network. Vu et al. in [11] revises the algorithm in [16], and shows that the revised DFC algorithm converges to a fixed point, which can reduce the outage of wireless users as well as the consumed transmit power, as compared to TPC algorithms. However, a DFC algorithm is still not able to use under our scenario: EMI-based multiple-priority admission control in a mobile hospital, since DFC algorithms are designed for admission control within a network in which all of users are in the same priority, while in our scenario, the users are in different classes of priority. The candidate users for removal in DFC are determined by their transmit power, values of target SINR and channel conditions, irrespective of their priorities. So a few high-priority users may be removed before the removal of low-priority users, and thus cannot guarantee the minimum outage of high-priority users (see the results in Fig. 4 and Fig. 6).

In view of the abovementioned analysis on TPC and DFC algorithms, we develop an algorithm which is a combination of TPC and DFC under the scenario of admission control of priority-based users under the scenario of mobile hospital. In this algorithm, high-priority users employ the TPC algorithm, while low-priority users employ the DFC algorithm. The iterative setting of transmit power for user $i$ in our proposed algorithm is shown as

$$
\begin{aligned}
& P_{i}(t+1)=\min \left\{\bar{P}_{i}, \frac{\hat{\theta}_{i}}{\theta_{i}(t)} P_{i}(t)\right\}, \text { if } i \in U_{H} \\
& P_{i}(t+1)=\left\{\begin{aligned}
\frac{\hat{\theta}_{i}}{\theta_{i}(t)} P_{i}(t), & \text { if } \frac{\hat{\theta}_{i}}{\theta_{i}(t)} P_{i}(t) \leq P_{i}^{t h} \text { and } i \in U_{L} \\
0, & \text { if } \frac{\hat{\theta}_{i}}{\theta_{i}(t)} P_{i}(t)>P_{i}^{t h} \text { and } i \in U_{L}
\end{aligned}\right.
\end{aligned}
$$

where $\bar{P}_{i}$ denotes the maximal level of the transmit power for user $i ; \hat{\theta}_{i}$ is the acceptable level of SINR to user $i ; P_{i}(t)$ and $\theta_{i}(t)$ represent the transmit power and level of SINR at itera-

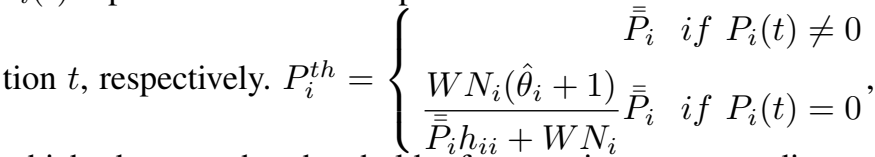
which denotes the threshold of transmit power to disconnect low-priority user $i^{2}$. $\overline{\bar{P}}_{i}=\min \left\{\bar{P}_{i}, \frac{\hat{\theta}_{i} R_{i} \sigma_{i}^{*}}{W h_{i i}}\right\}, \sigma^{*}=$ $\max _{i}\left\{S_{i}+W N_{i} \mid \frac{\hat{\theta}_{i} R_{i}\left(S_{i}+W N_{i}\right)}{W h_{i i}} \leq \bar{P}_{i}\right.$, for any $\left.i \in U_{H}\right\}$, and $S_{i}=\sum_{j} P_{j} h_{j i}$.

In the following section, we will show that the proposed algorithm in (9) can yield to the optimal solution to problem (8) while ensuring that EMI to all of medical sensors is at an acceptable level, i.e. the transmit power of all of wireless users is below METP (see Definition 1).

\section{ANALYSIS ON THE ALGORITHM OF POWER AND ADMISSION CONTROL}

\section{A. Preliminaries of analysis on the algorithm}

In this section, we present a few definitions to analyze the performance of proposed power and admission control algorithm.

\footnotetext{
${ }^{2}$ The reason of determining such a threshold is to guarantee the proposed algorithm (9) is Pareto-efficient and power-efficient, refer to Lemma 6 (part (c)) and Theorem 7
}

Definition 4: Let $U$ denote a set of users. Set $U$ is defined as accessible if for any user $i \in U$, the following inequality holds

$$
0 \leq f\left(\hat{\theta}_{i}, U\right) \leq \bar{P}_{i}
$$

where $\hat{\theta}_{i}$ is the acceptable level of SINR to user $i$, and $f\left(\hat{\theta}_{i}, U\right)$ is defined as (5) at $\theta_{i}=\hat{\theta}_{i}$.

Remark 4.1: A set $U$ is named as inaccessible if it is not accessible.

Definition 5: Let $U_{H}$ denote the set of high-priority users, while $U_{L}$ denotes the set of low-priority users. The set of $Q=U_{H} \cup Q_{L}$ where $Q_{L} \subset U_{L}$ is defined as Pareto-efficient if no low-priority user $i \notin Q$ and $Q \cup i$ is accessible.

Remark 4.2: A Pareto-efficient set indicates that no lowpriority users are allowed to admit into the network while keeping the network accessible. A set is named as Paretoefficiently accessible if and only if it is Pareto-efficient and accessible.

Definition 6: A set $U$ is Pareto-efficient and powerefficient, if it is Pareto-efficient and the transmit power $P_{i}$ reaches its minimum while meeting the requirements of SINR for any user $i \in U$.

The following theorems address the sufficient and necessary conditions of an accessible set as well as a Pareto-efficiently accessible set, respectively.

Theorem 2: The set of $Q=U_{H} \cup Q_{L}$ where $Q_{L} \subset U_{L}$ is accessible, if and only if each of low-priority users $i \in Q$, the following inequality holds:

$$
0 \leq f\left(\hat{\theta}_{i}, Q\right) \leq \min \left\{\bar{P}_{i}, \frac{\hat{\theta}_{i} R_{i} \sigma^{*}}{W h_{i i}}\right\}
$$

Proof: We first show that when inequality (10) holds, the set of $Q=U_{H} \cup Q_{L}$ is accessible.

When $i \in U_{H}, 0 \leq f\left(\hat{\theta}_{i}, Q\right) \leq \frac{\hat{\theta}_{i} R_{i} \sigma^{*}}{W h_{i i}} \Rightarrow$ $\frac{W N_{i}}{2\left(1-\sum_{k \in Q} \frac{R_{k} \theta_{k}}{W+R_{k} \theta_{k}}\right)} \leq \sigma^{*} \leq \frac{W \bar{P}_{i} h_{i i}}{\hat{\theta}_{i} R_{i}} \Rightarrow 0 \leq f\left(\hat{\theta}_{i}, Q\right) \leq \hat{P}_{i}$. When $i \in U_{L}$, inequality (10) indicates $0 \leq f\left(\hat{\theta}_{i}, Q\right) \leq \hat{P}_{i}$. Thus, $0 \leq f\left(\hat{\theta}_{i}, Q\right) \leq \hat{P}_{i}$ for $i \in Q$, i.e. the set of $Q$ is accessible.

Then, we show that the accessibility of $Q$ indicates $0 \leq$ $f\left(\hat{\theta}_{i}, Q\right) \leq \min \left\{\bar{P}_{i}, \frac{\hat{\theta}_{i} R_{i} \sigma^{*}}{W h_{i i}}\right\}$. When $i \in U_{H}$, we have

$$
\frac{W N_{i}}{2\left(1-\sum_{k \in Q} \frac{R_{k} \theta_{k}}{W+R_{k} \theta_{k}}\right)} \leq \sigma^{*} \text {. }
$$

Thus, $0 \leq f\left(\hat{\theta}_{i}, Q\right) \leq \frac{\hat{\theta}_{i} R_{i} \sigma^{*}}{W h_{i i}}$. When $i \in U_{L}$, we have $0 \leq$ $f\left(\hat{\theta}_{i}, Q\right) \leq \hat{P}_{i}$. So $0 \leq f\left(\hat{\theta}_{i}, Q\right) \leq \min \left\{\bar{P}_{i}, \frac{\hat{\theta}_{i} R_{i} \sigma^{*}}{W h_{i i}}\right\}$ for any $i \in Q$. The proof follows.

Theorem 3: The set of $Q=U_{H} \cup Q_{L}$ where $Q_{L} \subset U_{L}$ is Pareto-efficiently accessible, if and only if $Q$ is accessible and for any low-priority user $j \notin Q$, at least one low-priority user $i \in Q \cup\{j\}$ for which the following inequality does not hold:

$$
0 \leq f\left(\hat{\theta}_{i}, Q \cup\{j\}\right) \leq \min \left\{\bar{P}_{i}, \frac{\hat{\theta}_{i} R_{i} \sigma^{*}}{W h_{i i}}\right\}
$$

Proof: We first show that when at least one low-priority user $i \in Q \cup\{j\}$ for which inequality (11) does not hold, the set of $Q=U_{H} \cup Q_{L}$ is Pareto-efficiently accessible, given $Q$ is accessible. Let $i \in Q \cup\{j\}$ for which inequality (11) does not hold. 
When $\min \left\{\bar{P}_{i}, \frac{\hat{\theta}_{i} R_{i} \sigma^{*}}{W h_{i i}}\right\}=\bar{P}_{i}, f\left(\hat{\theta}_{i}, Q \cup\{j\}\right)>\bar{P}_{i}$, which indicates $Q \cup\{j\}$ is not accessible, so in such a case, the set of $Q=U_{H} \cup Q_{L}$ is Pareto-efficiently accessible. When $\min \left\{\bar{P}_{i}, \frac{\hat{\theta}_{i} R_{i} \sigma^{*}}{W h_{i i}}\right\}=\frac{\hat{\theta}_{i} R_{i} \sigma^{*}}{W h_{i i}}$, we have

$$
\frac{W N_{i}}{2\left(1-\sum_{k \in Q} \frac{R_{k} \theta_{k}}{W+R_{k} \theta_{k}}\right)}>\sigma^{*} \text {. }
$$

According to Theorem 2, $Q \cup\{j\}$ is not accessible, so the set of $Q=U_{H} \cup Q_{L}$ is Pareto-efficiently accessible.

Then, we show that the Pareto-efficient accessibility of $Q$ indicates at least one low-priority user $i \in Q \cup\{j\}$ for which inequality (11) does not hold. For any $j \notin Q$, the Paretoefficient accessibility of $Q$ indicates $Q \cup\{j\}$ is not accessible. Let $i \in Q \cup\{j\}$ and $f\left(\hat{\theta}_{i}, Q \cup\{j\}\right)>\bar{P}_{i}$.

When $i \in U_{L}, f\left(\hat{\theta}_{i}, Q \cup\{j\}\right)>\bar{P}_{i}$ indicates that $0 \leq$ $f\left(\hat{\theta}_{i}, Q \cup\{j\}\right) \leq \min \left\{\bar{P}_{i}, \frac{\hat{\theta}_{i} R_{i} \sigma^{*}}{W h_{i i}}\right\}$ does not hold. When $i \in$ $U_{H}, f\left(\hat{\theta}_{i}, Q \cup\{j\}\right)>\bar{P}_{i}$ indicates that

$$
\frac{W N_{i}}{2\left(1-\sum_{k \in Q} \frac{R_{k} \theta_{k}}{W+R_{k} \theta_{k}}\right)}>\sigma^{*} \text {. }
$$

Thus, for any $i \in Q \cup\{j\}$, we have $f\left(\hat{\theta}_{i}, Q \cup\{j\}\right)>\frac{\hat{\theta}_{i} R_{i} \sigma^{*}}{W h_{i i}}$, which also violates $0 \leq f\left(\hat{\theta}_{i}, Q \cup\{j\}\right) \leq \min \left\{\bar{P}_{i}, \frac{\hat{\theta}_{i} R_{i} \sigma^{*}}{W h_{i i}}\right\}$. The proof follows.

\section{B. Fixed-point of proposed algorithm}

Lemma 4: The iterative power-update function of the proposed algorithm (9) has at least one fixed point when $M_{1}=$ \{\} , i.e. DFC algorithm has at least one fixed point when all of users have the same priority.

Proof: Refer to Theorem 3 of [16].

Theorem 5: The iterative power-update function of the proposed algorithm (9) has at least one fixed point.

Proof: According to Lemma 4, DFC algorithm, which is employed by low-priority users, has at least one fixed point under any noise $N_{i}$. Since the transmit power of the high-priority users who employ TPC apprears as the noise to low-priority users, the iterative power-update function of the proposed algorithm (9) has at least one fixed point.

Lemma 6: For any fixed point $\mathbf{P}^{*}$ of the power-update function of the proposed algorithm (9), the following conditions hold.

(a) $\frac{W N_{i}}{2\left(1-\sum_{k \in S\left(\mathbf{P}^{*}\right)} \frac{R_{k} \theta_{k}}{W+R_{k} \theta_{k}}\right)} \leq \sigma^{*}$.

(b) For any user $i \in U_{H}, i \in S\left(\mathbf{P}^{*}\right)$ (see definition 1), i.e. the set of all of high-priority users is accessible.

(c) For any low-priority user $i \notin S\left(\mathbf{P}^{*}\right)$, we have $P_{i}^{*}=0$ and the following inequality does not hold:

$$
0 \leq f\left(\theta_{i}, S\left(\mathbf{P}^{*}\right) \cup\{i\}\right) \leq \overline{\bar{P}}_{i} .
$$

Proof: (a) If $\frac{W N_{i}}{2\left(1-\sum_{k \in S\left(\mathbf{P}^{*}\right)} \frac{R_{k} \theta_{k}}{W+R_{k} \theta_{k}}\right)}>\sigma^{*}$, then, $f\left(\theta_{i}, S\left(\mathbf{P}^{*}\right)>\frac{\sigma^{*} R_{i} \theta_{i}}{W h_{i i}} \geq \overline{\bar{P}}_{i}\right.$ for any low-priority user $i$, thus $P_{i}=0$ for any $i \in U_{L}$. Put it another way, all of low-priority users are disconnected from the network at the fixed point. Since the set of all of high-priority users is accessible, it indicates that $\frac{W N_{i}}{2\left(1-\sum_{k \in S\left(\mathbf{P}^{*}\right)} \frac{R_{k} \theta_{k}}{W+R_{k} \theta_{k}}\right)} \leq \sigma^{*}$, which leads to a contradiction. Thus, $\frac{W N_{i}}{2\left(1-\sum_{k \in S\left(\mathbf{P}^{*}\right)} \frac{R_{k} \theta_{k}}{W+R_{k} \theta_{k}}\right)} \leq \sigma^{*}$.

(b)Since $\frac{W N_{i}}{2\left(1-\sum_{k \in S\left(\mathbf{P}^{*}\right)} \frac{R_{k} \theta_{k}}{W+R_{k} \theta_{k}}\right)} \leq \sigma^{*}$, we can easily show that for any user $i \in U_{H}, i \in S\left(\mathbf{P}^{*}\right)$ by referring to the first part of proof of Theorem 2 .

(c) It is obvious that $P_{i}^{*}=0$ for any user $i \notin S\left(\mathbf{P}^{*}\right)$. Otherwise, $P_{i}^{*}=\frac{\hat{\theta}_{i}}{\theta_{i}} P_{i}^{*}$ (see (9)), so $\hat{\theta}_{i}=\theta_{i}$, implying $i \in$ $S\left(\mathbf{P}^{*}\right)$, which leads to a contradiction.

If inequality (12) holds, then, for any given low-priority user $i \notin S\left(\mathbf{P}^{*}\right)$, inequality (12) can be represented as

$$
0 \leq f\left(\theta_{i}, S\left(\mathbf{P}^{*}\right)\right) \leq \frac{N_{i}\left(\hat{\theta}_{i}+1\right)}{\overline{\bar{P}}_{i} h_{i i}+N_{i}} \overline{\bar{P}}_{i}
$$

which contradicts the constraint $f\left(\theta_{i}, S\left(\mathbf{P}^{*}\right)\right)>\frac{N_{i}\left(\hat{\theta}_{i}+1\right)}{\bar{P}_{i} h_{i i}+N_{i}} \overline{\bar{P}}_{i}$ of fixed point when $P_{i}=0$ (see (9)).

Theorem 7: Any fixed point of the iterative power-update function of the proposed algorithm (9) is Pareto-efficient and power-efficient.

Proof: Lemma 6 shows that $S_{H}(\mathbf{P})=U_{H}$. Also from lemma 6, we know that the inequality (12) does not hold. From Theorem 3, we conclude that the iterative power-update function of the proposed algorithm (9) is Pareto-efficient. Also lemma 6 shows that $P_{i}^{*}=0$ for any user $i \notin S\left(\mathbf{P}^{*}\right)$ and $\theta_{i}=$ $\hat{\theta}_{i}$ for any user $i \in S\left(\mathbf{P}^{*}\right)$. Thus, the iterative power-update function of the proposed algorithm (9) is power-efficient.

Lemma 8: When all of low-priority users have a common targeted SINR, the iterative power-update function of the proposed algorithm (9) provides a solution to the problem of minimizing outage ratio in (8) when $M_{1}=\{\}$, i.e. DFC algorithm provides a solution to the problem of minimizing outage ratio when all of users have the same priority and the common targeted SINR.

Proof: Refer to Theorem 6 of [16].

Theorem 9: When all of low-priority users have a common targeted SINR, any fixed point of the power-update function of the proposed algorithm (9) provides a solution to the problem of minimizing outage ratio in (8). Also at the fixed point of our proposed algorithm, the EMI to all of medical sensors is acceptable, i.e. the transmit power of all of wireless users is controlled below METP (see Definition 1).

Proof: Lemma 6 shows that all of high-priority users are supported with the proposed Algorithm of (9). So at most a few low-priority users are removed out of the network. Also according to lemma 8 , any fixed point of the power-update function of the proposed algorithm (9) provides a solution to the problem of minimizing outage ratio in (8) when all of users have the same priority and the common targeted SINR. According to (4), the interference caused by highpriority users appears as noise to low-priority users, so the proposed Algorithm of (9) provides a solution to the problem of minimizing outage ratio in (8) under the setting of twopriority users.

Also Theorem 7 indicates that the set of users which are not removed out of the network is accessible, and thus $0 \leq$ 
$P_{i} \leq \bar{P}_{i}$ holds by Definition 4 . So the transmit power of all of users is controlled below METP.

\section{PRACTICAL CONSIDERATIONS}

In this section, we address a few practical issues concerning the application of our power and admission control algorithm. These issues include a more general scenario of wireless users with more than two classes of priority as well as the modification of power and admission control algorithm when the system is inaccessible even after disconnecting all of lowpriority users.

\section{A. Multiple classes of priority}

In our proposed power and admission control algorithm, we assume that the set of users is composed of two classes of priority, which may not be true in a few actual scenarios. For example, the triage levels of emergency patients in a hospital are classified into 5 levels in Canada. When wireless users transmit the data of patients at different levels, the network administrator must firstly satisfy the data transmission of users who are sending the data of patients at the highest emergency level. Thus, the order of priority assigned to wireless users should be consistent with the emergency level of data which are sent by the users. In such scenarios, we first combine all classes of priority except the lowest priority into one priority, which is named as high priority, and name the originally lowest priority as low priority. Then, we can apply our proposed power and admission control algorithm into the high/low-priority scenario. If there is no solution available (i.e. the network is still not accessible even when all of lowest-priority users are disconnected), we can iteratively disconnect the lowest-priority ones among the high-priority users. This iterative strategy can guarantee the disconnection of minimal number of lowestpriority users and no disconnection of highest-priority users in a multiple-priority scenario. The detailed process of power and admission control is shown in Algorithm 1.

Under the setting of only two priorities, the proposed algorithm of (9) could function in a fully distributed manner. Under the setting of more priority classes, lower-priority users need network information to determine whether they belong to $U_{H}$ or not in a network with multiple priority classes. In this case, a network administrator would work to inform the users whether they belong to $U_{H}$ or not in a broadcasting way.

\section{B. Inaccessible network with the disconnection of all low- priority users}

In our proposed power and admission control algorithm, the existence of feasible solutions to problem (8) rests under the assumption that $U_{H}$ is accessible. Otherwise, a few highpriority users would be required to disconnect from the network in order to ensure that the set of left users in the network is accessible. Under the latter scenario, we need to modify our algorithm by attempting to disconnect high-priority users one by one. The order of disconnection refers to the value of $h_{i i}$ and $R_{i}$ of high-priority users. Such an order is determined by the TPC strategy which is employed by high-priority users. The detailed process of power and admission control is shown in Algorithm 2.

\begin{tabular}{l}
\hline Algorithm 1: Joint power and admission control under \\
$M$-priority scenario ( $M>2, U_{1}, \cdots, U_{M}$ represents the \\
set of users with priority ranking from high to low) \\
\hline Step 1: Combine the sets of users with priority \\
$U_{1}, \cdots, U_{M-1}$ into one set, denoted as $U_{H}$, i.e., \\
$U_{H}=U_{1} \cup \cdots \cup U_{M-1}$. Also we let $U_{L}=U_{M}$. \\
Step 2: Establish the problem of (8) and solve it using \\
power-update function in algorithm (9). \\
Step 3: If the problem of (8) has a solution, then, go to \\
step 4; Otherwise if $U_{H}=U_{1}$, go to step 5; Otherwise, \\
we remove all of users in $U_{L}$, recombine the sets of \\
users as $U_{H}=U_{1} \cup \cdots \cup U_{i-1}, U_{L}=U_{i}$ (where $U_{H}$ has \\
$i(i \leq M)$ classes of priority in such an iteration), and \\
then go to step 2. \\
Step 4: Return the solution to the problem of joint power \\
and admission control. \\
Step 5: Return the result of 'No feasible solution'. \\
\hline
\end{tabular}

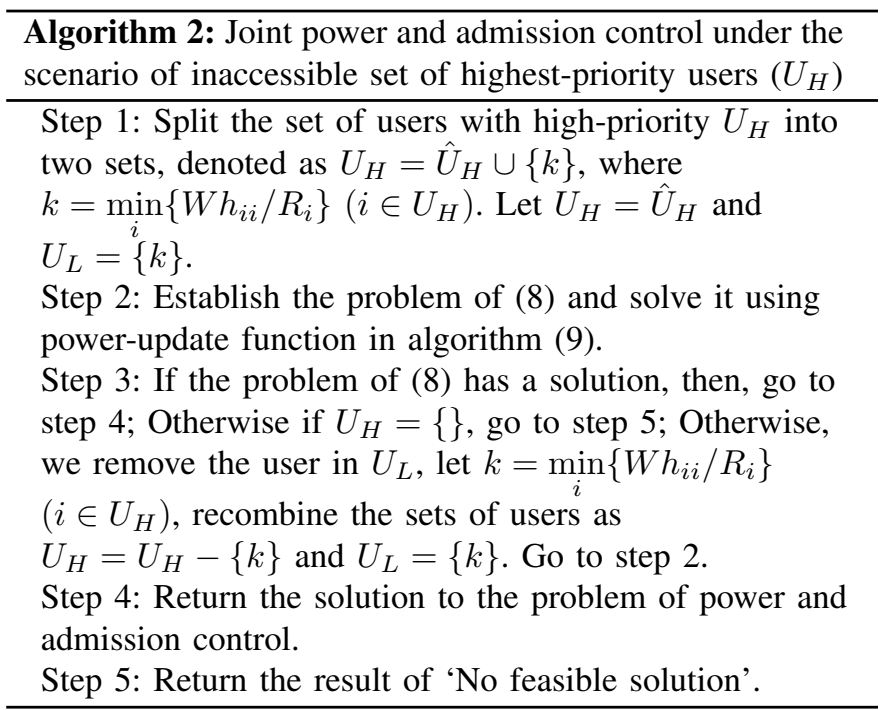

\section{Simulation RESUlts}

In all of simulation scenarios, we assume that a network administrator would work to gather the information of users, including the strength of signal power, the distance between mobile users and medical sensors, etc. with the aid of a few typical devices, such as signal strength meters, global positioning system (GPS) devices, and schedule the proposed algorithms based on the information. Also we assume that the mobile users are using a typical cellular network, such as universal mobile telecommunication system (UMTS) Network.

In the simulation, we consider a few typical empirical networks for Internet of vehicles, and a connection of network represents a transmit-receive pair of wireless users. Specifically, these networks include Erdös-Rényi network, Exponential network and preferential attachment network [18], and the distributions of their vertex degree are shown in Fig. 1. Each of vehicle networks contains 50 nodes, and each node has a probability of 0.1 using the mobile phone. Please note that in cities, when an ambulance is close to densely populated 

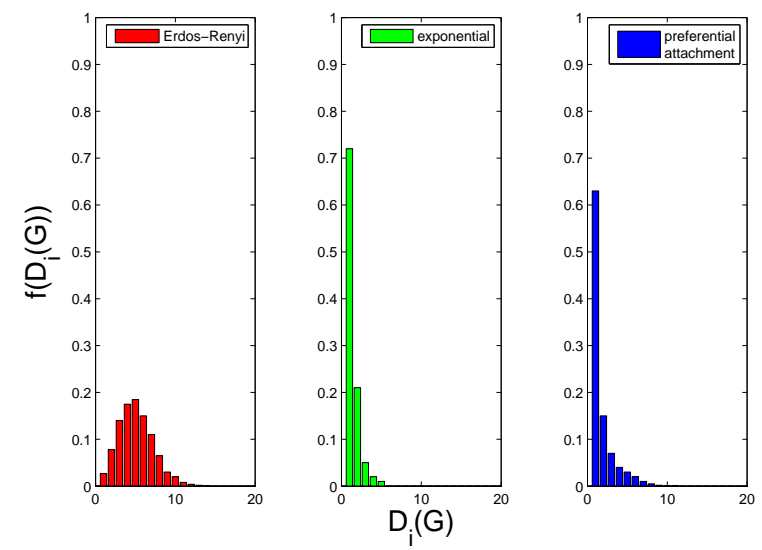

Fig. 1. The Figure illustrates representative vertex degree distributions for Erdös-Rényi (left) with $p=0.3$, Exponential (center) with $\alpha=2.5$, and preferential attachment (scale-free) graphs (right) with $\gamma=15 . f(D(G))$ is the frequency of the vertex degree $D(G)$.

areas, it is possible that 50 terminals have EMI impact on medical sensors at the same time. The average distance between terminals is 8 meters. Each terminal is moving with an arbitrary direction at a speed of $10 \mathrm{~m} / \mathrm{s}(36 \mathrm{~km} / \mathrm{h})$. Also we normalize the level of EMI $E_{L S}$ or $E_{N L S}$ (see (1) and (2)) to unity, and perform about 100000 Matlab-based experiments to present the results.

\section{A. Characteristics of channel models}

We select the commonly used set of empirical channel models, which is specified in ITU-R recommendation M.1225 [19], for simulation. ITU-R M.1225 model is applicable for the test scenarios in urban and suburban areas outside the high rise core where the buildings are of nearly uniform height [19]:

$$
L=40\left(1-4 \times 10^{-3} \Delta h\right) \log R-18 \log \Delta h+21 \log f+80
$$

where $R[\mathrm{~km}]$ represents the distance between base station and mobile station; $f[M H z]$ represents the carrier frequency; $\Delta h[m]$ represents the base station antenna height, which is measured from the average rooftop level.

Each terrestrial test environment can be modeled as a channel impulse response model based on a tapped-delay line. The model is characterized by the number of taps, the time delay relative to the first tap, the average power relative to the strongest tap, and the Doppler spectrum of each tap. A majority of time-delay spreads are relatively small, while a few "worst case" multipath characteristics cause much larger delay spreads. Table I identifies the propagation model for each of 6 vehicular test cases. In all of these test cases, we consider the strength and relative time delay of signal components as well as Doppler shift, and assume that each of 6 vehicular test cases occurs with the same probability. Specifically, the primary parameters to characterize each of propagation models include:

- Time delay-spread, its structure, and its statistical variability (e.g. probability distribution of time delay spread);

- Multipath fading characteristics (e.g. Doppler spectrum, Rician vs. Rayleigh) for the envelope of channels.

\begin{tabular}{|c|c|c|c|}
\hline \hline Tap & $\begin{array}{c}\text { Relative } \\
\text { delay }(\mathrm{ns})\end{array}$ & $\begin{array}{c}\text { Average } \\
\text { power }(\mathrm{dB})\end{array}$ & $\begin{array}{c}\text { Doppler } \\
\text { spectrum }\end{array}$ \\
\hline 1 & 0 & 0.0 & Rayleigh \\
\hline 2 & 310 & -1.0 & Rayleigh \\
\hline 3 & 710 & -9.0 & Rayleigh \\
\hline 4 & 1090 & -10.0 & Rayleigh \\
\hline 5 & 1730 & -15.0 & Rayleigh \\
\hline 6 & 2510 & -20.0 & Rayleigh \\
\hline
\end{tabular}

TABLE I

PARAMETERS OF PROPAGATION MODELS IN ITU-R RECOMMENDATION M.1225 [19]

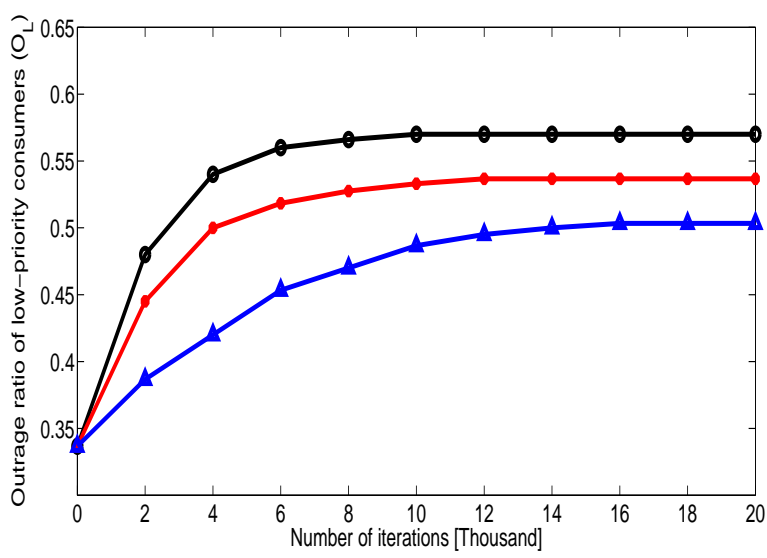

Fig. 2. The Figure illustrates the rate of convergence to the fixed point of our algorithm under different random networks. Blue line with ' $\triangle$ ' represents Exponential network; Red line with ' ' represents preferential attachment (scale-free) network; Dark line with 'o' represents Erdös-Rényi network.

\section{B. Convergence of proposed algorithm}

In this section, we compare the convergence rate of our algorithm (9) under the scenarios of different random networks. For simplicity, we set the target SINR of each information user as $10 \mathrm{~dB}$, and investigate the convergence rate to the target SINR for different networks.

It is observed from Fig. 2 that the algorithm of (9) under the networks with highly concentrated transmit/receive nodes (e.g., Exponential network) quickly converges to the fixed point ${ }^{3}$, while the algorithm under the networks without highly concentrated transmit/receive nodes (e.g., Erdös-Rényi network) converges to the fixed point at a low rate. Indeed, the algorithm under the Exponential network reaches the fixed point after 7000 iterations, while its convergence appears after 12000 iterations under the Erdös-Rényi network.

This is because a user establishs transmit-receive pairs with most of the other users in Erdös-Rényi network, and thus one data transmission is easily influenced by the interference from

\footnotetext{
${ }^{3}$ With the Intel Core $17-2760 \mathrm{QM}$ processor, the running time of each iteration is around $0.00014 \mathrm{~s}$, so the total time of running the algorithm with 6000 iterations is $0.84 \mathrm{~s}$. Given that the ambulance is moving at a speed of $10 \mathrm{~m} / \mathrm{s}$, the algorithm is feasible when the channel conditions are assumed to be invariant within a distance of $8.4 \mathrm{~m}$. In a fast-varying mobile environment, we can use a more powerful processor to run the algorithm to ensure its feasibility.
} 


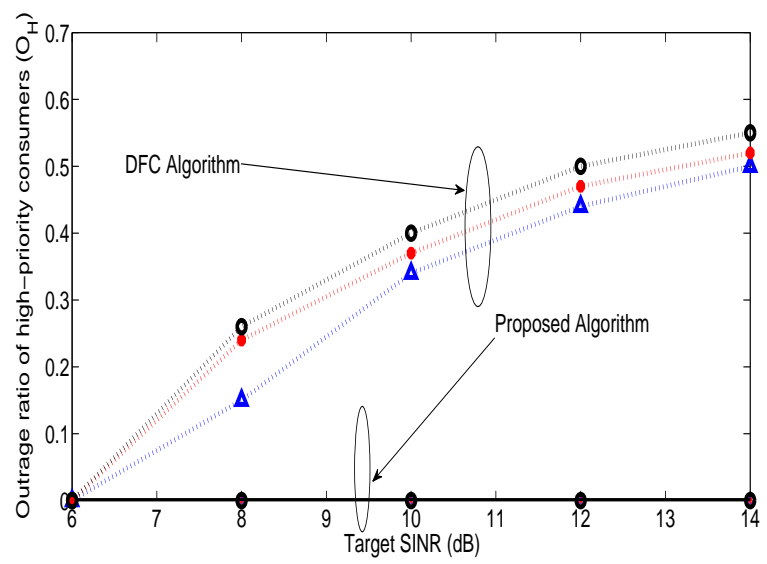

Fig. 3. The Figure illustrates the outage ratio of high-priority users with various values of target SINR (dB) under different random networks (Proposed algorithm vs. DFC algorithm [11]). Blue line with ' $\triangle$ ' represents Exponential network; Red line with ' ' represents preferential attachment (scale-free) network; Dark line with 'o' represents Erdös-Rényi network.

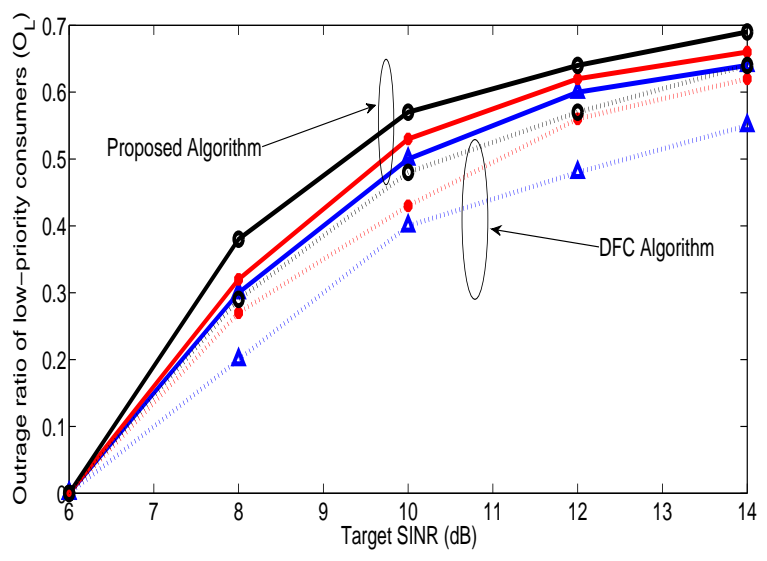

Fig. 4. The Figure illustrates the outage ratio of low-priority users with various values of target SINR (dB) under different random networks (Proposed algorithm vs. DFC algorithm [11]). Blue line with ' $\triangle$ ' represents Exponential network; Red line with '.' represents preferential attachment (scale-free) network; Dark line with 'o' represents Erdös-Rényi network.

the other transmissions. However, in the Exponential network, the users establish transmit-receive pairs with only a single or few other users, and they suffer little interference from the other transmissions.

\section{Outage ratio of users}

It is observed from Fig. 3 that our proposed algorithm can always guarantee a zero outage of high-priority users under various networks, while the DFC algorithm cannot guarantee a zero outage. Instead, the outage of high-priority users by DFC reaches up to $50 \%$ when the target SINR of all users is set as $14 \mathrm{~dB}$. However, as shown in Fig. 4, DFC can attain a lower outage ratio for low-priority users, in comparison with our proposed algorithm. This is because DFC algorithm cannot guarantee zero-outage for high-priority users. To ensure zero outage of high-priority users, the users who disconnect from

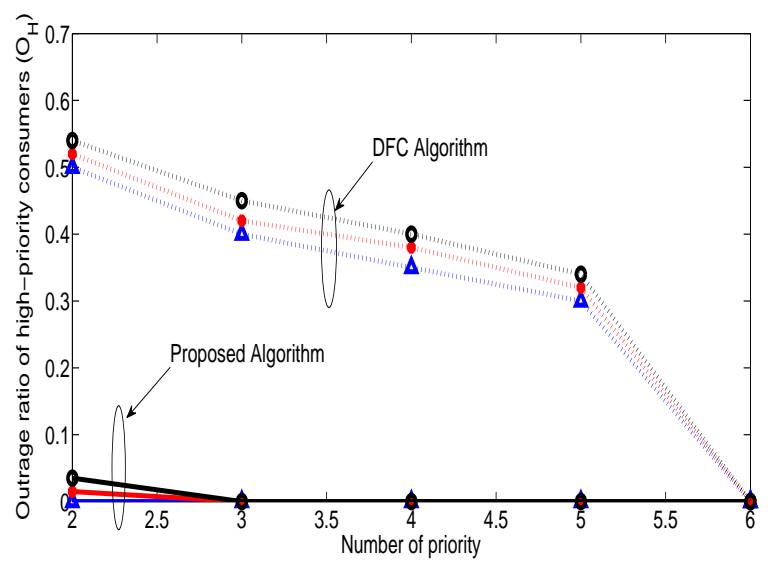

Fig. 5. The Figure illustrates the outage ratio of high-priority users vs. number of priority under different random networks. Blue line with ' $\mathrm{o}$ ' represents Exponential network; Red line with ' ' represents preferential attachment (scale-free) network; Dark line with ' $\triangle$ ' represents Erdös-Rényi network.

network can only be chosen among low-priority users, as the case of our proposed algorithm.

Another result observed from Fig. 3 and Fig. 4 is that lower outage ratio can be achieved by Exponential network, in which wireless users have only a single or few transmit/receive pairs, than by Erdös-Rényi network in which users have multiple transmit/receive pairs. This is because wireless users in the network with highly concentrated transmit/receive nodes (e.g., Exponential network) would suffer a low level of interference.

\section{Setting of multiple classes of priority}

Fig. 5 shows the application of proposed modified algorithm (Algorithm 1) into a more general setting in which the network is composed of multiple-priority users. Also we set the target SINR as $14 \mathrm{~dB}$, and evenly divide the users into each class of priority. In this setting, the outage ratio of highest-priority users decreases with the number of classes of priority by both our algorithm and by DFC. The number of highest-priority users is $1 / K$ of total number of users in the network for $K$ priority. With the rise of $K$, the set of highest-priority users is inclined to be accessible, so the outage ratio decreases with $K$. Again, our algorithm can attain a much lower outage ratio of highest-priority users than DFC algorithm, which is in line with the results in Section VI.B.

However, different from the results of 2-priority setting in Section VI.B., an interesting result is that our algorithm cannot guarantee a zero outage ratio of highest-priority users when the target SINR is $14 \mathrm{~dB}$. This is because in such a scenario the set of all highest-priority users is not accessible, and thus a few of these users must be required to disconnect from the network (see Algorithm2). Also when the number of priority increases to 3 , the set of highest-priority users becomes accessible, and thus the outage ratio by our algorithm again reaches to zero.

\section{E. EMI to medical sensors}

In the following, we address the benefits of using the proposed algorithm to the decrease of EMI on medical sensors 

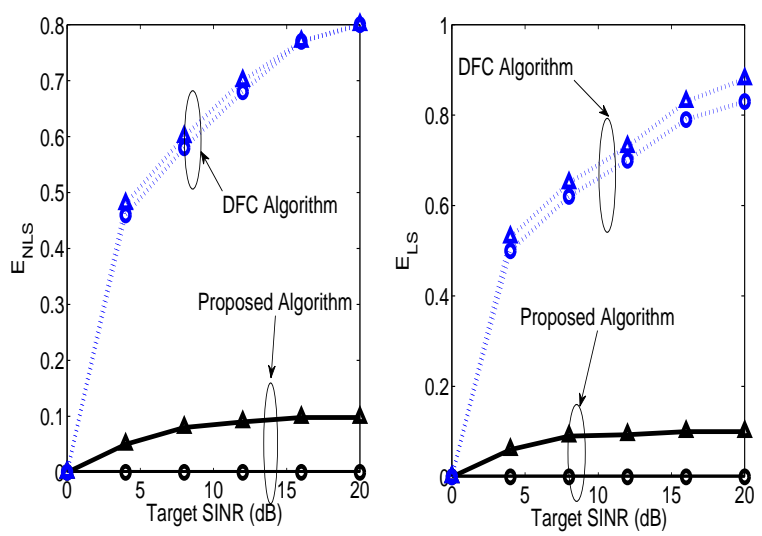

Fig. 6. The Figure shows EMI caused by RF transmission using our proposed algorithm vs. DFC algorithm: the left shows the EMI on non-life-support sensors, while the right shows the EMI on life-support sensors. Blue and dashed line represents using algorithm in [11]; Dark and solid line represents using our algorithm. Line with ' $\triangle$ ' represents the case of $m_{1}+m_{2}>n$; Line with 'o' represents the case of $m_{1}+m_{2} \leq n$.

under an Exponential network ${ }^{4}$. Fig. 6 shows the comparison of EMI on medical sensors caused by RF transmission between using our proposed algorithm (see (9)) as well as using the DFC algorithm proposed in [11]. Fig. 6 implies that our proposed algorithm (EMI level below 0.1 ) can dramatically reduce the amount of EMI on medical sensors than the algorithm in [11] (EMI level up to 0.8). Also our algorithm can ensure that medical sensors are free from EMI when $m_{1}+m_{2} \leq n$, and can ensure the minimal amount of EMI when $m_{1}+m_{2}>n$. Put it another way, when we need to consider the EMI on a large number of medical sensors $\left(m_{1}+m_{2}>n\right)$, our algorithm can minimize the amount of EMI on medical sensors though it cannot keep medical sensors free from EMI as under the scenario of a small number of medical sensors $\left(m_{1}+m_{2} \leq n\right)$.

\section{CONCLUSION}

We address a joint power and admission control algorithm to schedule the data transmission by wireless users over Internet of vehicles, so that the outage of high-priority users can be reduced to zero if the set of high-priority users is accessible and the outage ratio of low-priority users can be minimized. Also a few practical considerations in the applications of our algorithm under general scenarios are discussed. Some of the key inferences drawn are

- Proposed priority-based power and admission control algorithm can dramatically reduce the EMI on medical sensors as well as the outage ratio of users than DFC algorithm, which is the most widely-used admission control algorithm under the non-priority scenario.

- Under the networks with users who have highly concentrated transmit/receive pairs, the priority-based power and admission control algorithm can converge to the fixed point at a higher rate than under the networks in which transmit/receive pairs are evenly distributed among wireless users.

- Networks with users who have highly concentrated transmit/receive pairs can achieve a lower outage ratio (i.e., suffer

\footnotetext{
${ }^{4}$ The EMI results under the other networks are similar, so we only show the results under a single network.
}

less interference) than the networks in which transmit/receive pairs are evenly distributed among wireless users .

We would like to extend our results to a dynamic setting, in which the structure of Internet of vehicles might keep changing over time. Under such a dynamic setting, we would like to investigate how to design joint power and admission control algorithms.

\section{REFERENCES}

[1] P. Phunchongharn, D. Niyato, E. Hossain and S. Camorlinga. An EMIAware Prioritized Wireless Access Scheme for e-Health Applications in Hospital Environments, IEEE transactions on information technology in biomedicine, 14(5): 1247-1258, 2009.

[2] P. Phunchongharn, E. Hossain, S. Camorlinga. Electromagnetic Interference-Aware Transmission Scheduling and Power Control for Dynamic Wireless Access in Hospital Environments. IEEE Transactions on Information Technology in Biomedicine, 15(6): 890-899, 2011.

[3] Furahata. Electromagnetic interferences of electric medical equipment from hand-held radiocommunication equipment. International Symposium on Electromagnetic Compatibility, 468-471, 1999.

[4] Qinghua Shen, Xiaohui Liang, Xuemin Shen, Xiaodong Lin, H.Y Luo. Exploiting Geo-Distributed Clouds for a E-health Monitoring System With Minimum Service Delay and Privacy Preservation. IEEE Journal of Biomedical and Health Informatics, 18(2): 430 - 439, 2004.

[5] M. Hayajneh and C. T. Abdallah. Distributed joint rate and power control game-theoretic algorithms for wireless data. IEEE Communications Letter, 8(8): 511-513, 2004.

[6] Saul G Myerson. Mobile phones in hospitals are not as hazardous as believed and should be allowed at least in non-clinical areas. British Medical Journal, 326(7387): 460-461, 2003.

[7] Fiori, Franco. Integrated Circuit Susceptibility to Conducted RF Interference. Compliance Engineering, 2014.

[8] Giovanni Acampora, Diane J. Cook, Parisa Rashidi, and Athanasios V. Vasilakos. A Survey on Ambient Intelligence in Healthcare. Proceedings of the IEEE, 101(12): 2470-2494, 2013.

[9] Daojing He, Chun Chen, Sammy Chan, Jiajun Bu, Athanasios V. Vasilakos. ReTrust: Attack-Resistant and Lightweight Trust Management for Medical Sensor Networks. IEEE Transactions on Information Technology in Biomedicine. 16(4): 623-632, 2012.

[10] Naixue Xiong, Athanasios V. Vasilakos, Laurence Tianruo Yang, Lingyang Song, Yi Pan, Rajgopal Kannan, Yingshu Li. Comparative analysis of quality of service and memory usage for adaptive failure detectors in healthcare systems. IEEE Journal on Selected Areas in Communications, 27(4): 495-509, 2009.

[11] Vu Nguyen Ha, Long Bao Le. Distributed Base Station Association and Power Control for Heterogeneous Cellular Networks. IEEE Transactions on Vehicular Technology, 63(1): 282-296, 2014.

[12] Shih-En Wei, Hung-Yun Hsieh, Hsuan-Jung Su. Joint optimization of cluster formation and power control for interference-limited machine-to-machine communications. Global Communications Conference (GLOBECOM): 5512 - 5518, 2012.

[13] G. J. Foschini and Z. Milijanic. A simple distributed autonomous power control algorithm and its convergence. IEEE Transactions on Vehicular Technology, 42: 641-646, 1993.

[14] Ya-Feng Liu, Yu-Hong Dai. Joint power and admission control via $\mathrm{p}$ norm minimization deflation. IEEE International Conference on Acoustics, Speech and Signal Processing (ICASSP): 4789 - 4793, 2013.

[15] F. Berggren, R. Jantti, and S. Kim. A generalized algorithm for constrained power control with capability of temporary removal. IEEE Transactions on Vehicular Technology, 50(6): 1604-1612, 2001.

[16] M. Rasti, A. R. Sharafat, and J. Zander. Pareto and energy-efficient distributed power control with feasibility check in wireless networks. IEEE Transactions on Information Theory, 57(1): 245-255, 2011.

[17] Yingsong Huang, Shiwen Mao. Downlink Power Control for MultiUser VBR Video Streaming in Cellular Networks. IEEE Transactions on Multimedia, 15(8): 2137 - 2148, 2013.

[18] Jacomo Corbo, Di Lin. Optimal Pricing with Positive Network Effects: The Big Benefits of Just a Little Discrimination. International Conference on Information Systems, 2012.

[19] ITU-R Recommendation M.1225. Guidelines for evaluation of radio transmission technologies for IMT-2000, 1997. 